\title{
Caligrafía y calibre del WDWJJIR Los grupos cooperativos para el aprendizaje de sistemas de texturización óptica en procesos de reintegración formal y cromática en obras de arte
}

\author{
Antoni Colomina Subiela ${ }^{a}$ y Vicente Guerola Blay ${ }^{b}$ \\ a Instituto de Restauración del Patrimonio de la Universitat Politècnica de València; ancosu@upvnet.upv.es \\ b Instituto de Restauración del Patrimonio de la Universitat Politècnica de València; vguerola@crbc.upv.es
}

\begin{abstract}
\$EWWFW
$7 W W A J J R$, as a reintegration system of losses or missing parts of a pictorial o polychrome work, was used initially in the middle of the last century in different centres in Italy, rapidly spreading to the countries influenced by the Italian culture. Nowadays, this retouching technic, which has as an objective the differentiation of the restored parts through a graphic-optical texturing formula, is introduced as a discipline in the undergraduate and master's study programme in conservation and restoration at the national level. The need for learning, which we could consider as the calligraphy of the WDWJJ JIR, allows the students and the future conservation and restoration professionals to practice this discipline, making it necessary to regulate some standards of LCTD variables (length, contour, translucency and dispersion) too. The purpose of this study is to define standardization processes and cooperative group work guidelines where the participation of different professionals is required, as well as the introduction of a measurer of WDWAJ JIR as a prototype for implementation in studies of conservation and restoration of cultural heritage.
\end{abstract}

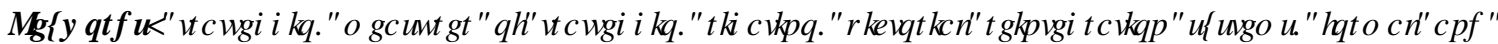

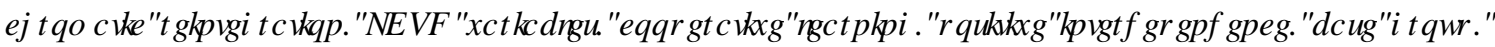
FRQWUXFWHFRQWRYHWI

\section{HXP HQ}

El WDWAJ IR como sistema de reintegración de lagunas o partes faltantes de una obra pictórica o polícroma fue puesto en marcha a mediados del siglo pasado en diferentes centros de restauración en Italia, extendiéndose rápidamente a los países de su arco de influencia cultural. Actualmente, esta técnica, que tiene como objetivo la diferenciación o discernibilidad de las partes intervenidas a través de una fórmula de texturización gráfico-óptica, se encuentra introducida como disciplina en los planes de estudio de grado y máster en conservación y restauración a nivel nacional. La necesidad de aprendizaje, de la que podríamos considerar como la caligrafía del WDWJJIR, que permita a los estudiantes y en un futuro profesionales de la conservación y restauración la práctica de esta disciplina, hace necesaria también la normalización de unos estándares de variables LCTD (longitud, contorno, traslucidez y dispersión). La finalidad del presente estudio es acotar procesos de estandarización y pautas de trabajo grupal cooperativo donde se requiera la participación de diferentes profesionales, así como, la introducción de un sistema de medición del WDWAJ JIR como prototipo para la implementación en los estudios de conservación y restauración de bienes culturales.

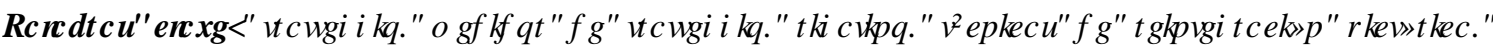

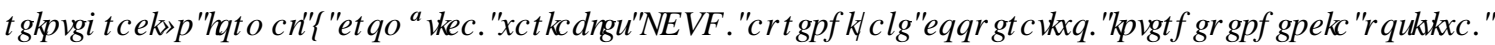
JUXSRIGHEDMHIFRQWRYHUDFRQMUXWWDD 


\section{Introducción}

El WWWJJIR es un sistema utilizado para la reintegración formal y cromática de partes faltantes en una pintura o superficie polícroma. Esta técnica está basada principalmente en la utilización de una trama gráfico-óptica a base de rayas que, interpuesta en el original, permite al espectador visualizar y reconocer la parte restituida, siendo imperceptible conforme nos alejemos de la visión de proximidad. Tratar de escalar o calibrar el formato del WDWAJ JIR es una tarea fundamental a la que, hasta la fecha, se le ha dado muy poca trascendencia y que nosotros hemos tratado de extrapolar a través de un estudio de variables que condicionan esta técnica. Sin lugar a dudas, el tamaño de la obra, bien se trate de una miniatura o de un lienzo de gran formato; su ubicación o distancia a la que va a ser observada, bien como pintura de caballete o como un gran fresco; y los condicionantes propios de la obra expuesta en un museo o formando parte de un espacio sacro; en definitiva, el formato, extensión y ubicación de las pérdidas o lagunas, forman parte de una extraordinaria casuística que condicionará el sistema, la caligrafía y el calibre del WDWAJ JIR.

Cuando se utiliza en trabajos de gran envergadura que requieren de la participación de varios restauradores, es necesario consensuar unos patrones de estandarización que permitan un tratamiento homogéneo en su realización, independientemente de la mano particularizadora que desarrolle el proceso. En este estudio se aborda la instrumentalización de un innovador “medidor de WDWJJ JR', desarrollando pedagógicamente un modelo de aprendizaje grupal cooperativo en vistas a la activación de esta práctica de reintegración textural.

Esta innovación ha sido aplicada dentro de la programación docente de la asignatura "Técnicas de reintegración cromática en bienes culturales", perteneciente al Grado de Conservación y Restauración de Bienes Culturales de la Universitat Politècnica de València, durante el segunto semestre de los cursos académicos 2017-2018 y 2018-2019 El número de alumnos de clase en la asignatura es aproximadamente de 32, de modo que para el desarrollo de esta actividad se establecieron 8 grupos de trabajo, formados cada uno por 4 personas. La asignatura se distribuye en un total de 6 créditos ECTS, con 10 horas de teoría de aula y 50 horas de prácticas de laboratorio. Del tiempo dedicado a las prácticas se destinaron 12 horas para las sesiones de trabajo de esta innovación, lo que supone, de acuerdo con la tabla 1, un cronograma que se extiende durante tres semanas, con 2 clases de 2 horas por semana.

\begin{tabular}{|c|c|c|}
\hline \multicolumn{2}{|c|}{ Temporalización } & Distribución \\
\hline \multirow[b]{2}{*}{ Semana 1} & Sesión 1 & $\begin{array}{l}30 \text { min: reunión de grupos informales } \\
90 \text { min: trabajo en grupos formales }\end{array}$ \\
\hline & Sesión 2 & $\begin{array}{l}30 \text { min: reunión de grupos informales } \\
30 \text { min: exposición general por parte de los grupos informales } \\
60 \text { min: trabajo en grupos formales }\end{array}$ \\
\hline \multirow[t]{2}{*}{ Semana 2} & Sesión 3 & $\begin{array}{l}105 \text { min: trabajo en grupos formales } \\
15 \text { min: reunión de grupos de base }\end{array}$ \\
\hline & Sesión 4 & 120 min: trabajo en grupos formales \\
\hline \multirow[t]{2}{*}{ Semana 3} & Sesión 5 & $\begin{array}{l}15 \text { min: reunión de grupos de base } \\
105 \text { min: trabajo en grupos formales }\end{array}$ \\
\hline & Sesión 6 & 120 min: trabajo en grupos formales \\
\hline
\end{tabular}




\section{Objetivos}

El proceso de reintegración cromática persigue completar la continuidad para recuperar la legibilidad de la imagen perdida por la aparición de lagunas de color que imposibilitan la contemplación y comprensión de las obras de arte (Calvo, 1997: 188-189). El ajuste cromático de estas pérdidas atiende a la disposición de trazos discernibles que componen la nueva adición del color. Estas intervenciones de restauración, a menudo, precisan de varios operadores que trabajen bajo una misma pauta gráfica, de modo que, el objetivo principal de esta propuesta de innovación docente es el establecimiento consensuado de una norma que codifique diferentes opciones de caligrafía y calibre del trazo de reintegración.

Para llevar a término este propósito general se establecen los siguientes objetivos específicos:

- Establecer grupos-clase para que convengan un medidor de WDWJJIR que sirva como estándar normalizado a través del trabajo cooperativo.

- Ejercitar con los alumnos la práctica del proceso de reintegración cromática considerando las variables de longitud, contorno, translucidez y dispersión del trazo gráfico.

- Reforzar el aprendizaje cooperativo con la creación de grupos informales y grupos de base para fomentar el desarrollo interpersonal y la socialización entre estudiantes.

- Reconocer la importancia de la interdependencia positiva en los grupos de trabajo y dinamizar las tareas por medio de los debates constructivos de controversia.

\section{EI WDWWJIR como procedimiento artístico y su adapatación técnica al campo de la reintegración cromática.}

El WDWJ JIR ha venido utilizándose desde antiguo y de forma continua como base de diferentes expresiones artísticas, tomando etimológicamente el vocablo del idioma italiano. 7WWJJIR son las líneas trazadas en paralelo para configurar el volumen o claroscuro en un dibujo. Esta técnica de gesto más o menos libre y espontáneo permite como solución gráfica alcanzar la conformación del objeto representado a través de la superposición de trazos en paralelo y entrecruzados para coseguir la imagen deseada. Algo semejante sucede con el grabado en muchas de sus variantes procedimentales, al conseguir a través de pequeñas y finas líneas en paralelo efectos de gradación tonal. Como $\amalg W W J J I R$ se podrían definir las propias pinceladas sutiles, paralelas y amalgamadas utilizadas en la técnica del temple de huevo (figura 1), que tan extraordinarias obras propició en el periodo gótico. Ese mismo concepto de líneas se desarrolla en la técnica del fresco a través de pinceladas, de nuevo paralelas, en la dirección del modelado de las formas, pero esta vez, a través de pinceles de notable extensión y dureza que permiten abordar superficies de mayor empeño. Esta fórmula de bosquejo rayado se puede apreciar en la superficie del modelado en barro a través de diferentes herramientas o con las gradinas y cinceles en el tallado de la piedra, ambos ejemplos en los procesos anteriores al alisado o pulido de la obra escultórica. En definitiva, WDWJJIR, que procede del vocablo WDWR en italiano (Tam, 2009), significa trazo; y WDWJIDH como verbo transitivo, se traduciría como la acción de trazar, aspecto que se desarrolla en el proceso gráfico de dibujar, grabar, pintar, modelar o esculpir.

El otro término común a la reintegración y también utilizado de forma usual en el vocabulario del restaurador es\UJDMQR, que se corresponde exactamente con la misma acepción de WDWJJIR(Calvo, 1997: 224) $\square$ si bien, en este caso, etimológicamente "riga" en italiano significa raya. Técnica del rayado o del trazado sería la forma correcta de traducir esta fórmula de texturización óptica utilizada como trama diferenciadora en procesos de retoque de obras de arte de carácter pictórico o polícromo. 
[

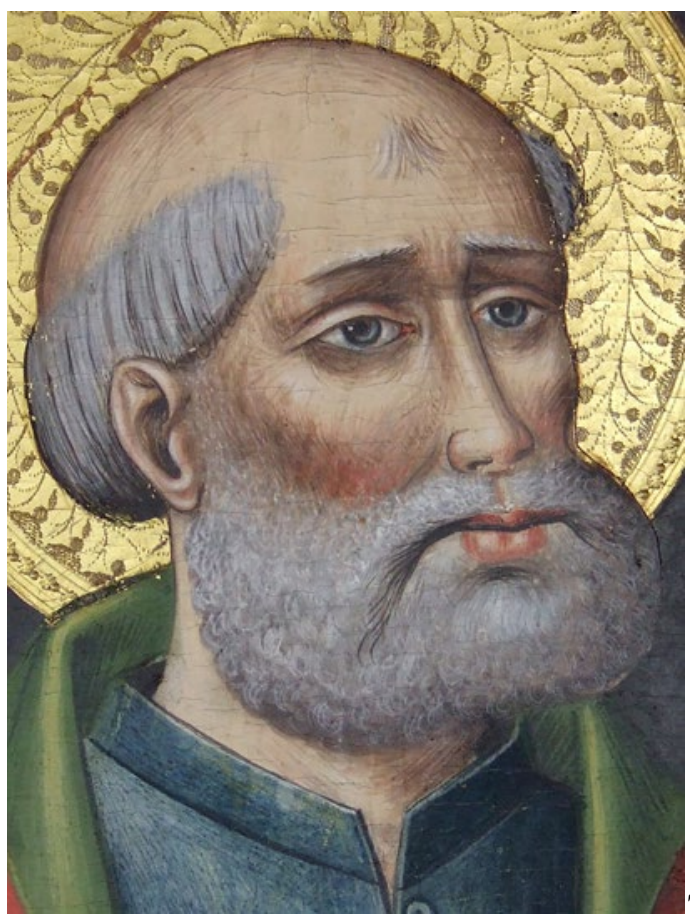

[

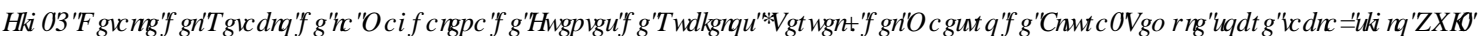

\section{Aproximación y valoración crítica del WDWEJJR}

El tratamiento de pérdidas y faltantes en obras de arte ha sido objeto de una especial atención, debate y controversia a partir de la segunda mitad del siglo pasado (Conzález-Varas, 1999: 122-126), dentro de los procesos de la reconocida como restauración en su proceso de intervención estética. Esta disciplina, también conocida como retoque pictórico, se ha movido generalmente entre dos polos radicalmente opuestos, basados en la discernibilidad o velado de su proceso de ejecución y puesta en práctica. Generalmente, aunque no se pueda generalizar, estas dos opciones han sido defendidas desde foros o ámbitos profesionales diferentes (Macarrón y González, 2007: 116-124). Por una parte, el espacio más académico y formativo, defensor, prácticamente inamovible, del reconocimiento de las zonas intervenidas a través de códigos basados principalmente en el uso del WDWJ JIR y sus variantes; en oposición, por otro lado, al mercado del arte y los anticuarios, que rechazan de plano lo que ellos consideran una manifestación de retoque ajena e hiriente con el original. Estas dos posturas enfrentadas esconden, sin lugar a dudas, intereses desde su esfera profesional, si bien los límites y márgenes en el proceso de reintegración de obras de carácter pictórico y policromo distan, en muchos casos, de las posiciones defendidas por una u otra opción, como trataremos de manifestar más adelante. Parece, cuanto menos evidente y lógico, que los conservadores-restauradores plegados en su formación académica reivindiquen de forma estricta su posición de juicio ante esta disciplina de su plena incumbencia; mientras que el sector profesional del mercado, que mueve, no olvidemos, un considerable volumen económico, se incline por soluciones de retoque no discernible, manifestando en su defensa lo inapropiado de una adición divergente con el original.

Ambas posturas opuestas se diluyen en muchos casos y las defensas de una u otra opción acaban por encontrase ante la casuística que presentan particularmente muchas obras. Pongamos por caso una pintura que muestre infinidad de pequeñas pérdidas producidas por una micropulverulencia o diminutos faltantes en una obra sobre lienzo debido a la fatiga en el tejido. En estos casos resulta totalmente imposible llevar a 
término una reintegración discernible, ya que el pequeño tamaño de las lagunas queda totalmente colmatado por la acción del retoque. En el extremo opuesto tendríamos aquellas obras en las que la operación de reintegración sería totalmente desestimable, bien por tratarse de trabajos inacabados por su autor, bien por piezas donde la extensión de la pérdida no permite una reintegración lógica y satisfactoria. Este caso paradigmático de no intervención acuñado en Francia recibe el nombre de QHUDQIDUH(Bergueon, 1992).

En su 7HUDGHQ 5HWXWFIy Cesare Brandi (1995: 71-76) ya definió como capital el estudio del tratamiento de las lagunas partiendo de una base teórica y desestimando la fórmula empírica con la que generalmente se venía tratando esta disciplina. A él y, más concretamente, a las iniciativas desarrolladas desde el , WHALRI \&HQWDOHSHUIO5 HMDXUR de Roma se debe la puesta en marcha de los primeros ejemplos de la utilización del WDWJJ JIR como sistema y técnica de retoque discenible (Catalano, 1998: 42-54). Su testimonio será más tarde recogido por Umberto Baldini $(1978 ; 1981)$ y llevado a una particular caligrafía textural, en este caso divulgada a través de los trabajos llevados a término en el 2 SHIFIRJGHOH了 IHMH' XUH de Florencia. En un primer momento, Brandi definió como LIIDP HQMla fórmula del trabajo de texturización a través de un rayado continuo semejante, como él dijo, al tejido de un tapiz. Esta fórmula tipológicamente romana fue adaptada según una nueva variante en Florencia al plantear un WDWJJIR de líneas discontinuas a partir de trazos sueltos y superpuestos. A tenor de la nomenclatura utilizada por ambas escuelas debemos entender, como se ha señalado anteriormente, como una misma acepción los términos de WDWJJIR y UJDMQR, si bien en la práctica los sistemas de una y otra escuela son divergentes.

En la actualidad, los sistemas de reintegración a partir de tramas de texturización óptica han sido consensuadas y aceptadas de forma prácticamente internacional, si bien es cierto que se han abierto soluciones en orden a diferentes propuestas menos rígidas de aquellas establecidas desde los inicios de la aparición de estas fórmulas de reintegración. En estos momentos podemos encontrar posturas más intervencionistas respecto de las que dieron origen a postulados de naturaleza arqueológica, siendo revisadas muchas intervenciones llevadas a término a finales del siglo XIX y principios del XX. Se ha comprobado qué es y qué no es susceptible de ser reconstituido y han sido admitidas, en algunos casos, soluciones de tratamientos de reintegración de elementos estandarizables en beneficio de la legibilidad de la obra o de incorporación de elementos $H[Q R Y R$ que faciliten la lectura de estructuras ornamentales.

Como ya se ha señalado, de la rigidez encorsetada de las propuestas del WDWAJ JIR vertical de mediados del siglo XX se ha pasado a una ampliación de soluciones y variables, principalmente dirigidas a romper con la monodireccionalidad de propuestas excesivamente herméticas. Ahora podemos encontrar "WDWAJ JIR entrecruzado", semejante a la trama de un tejido de tafetán; "WDWJJIR multidireccional", que facilita la interacción de la trama respecto del original circundante a la laguna; así como muchas otras soluciones de trama textural que bautizaremos genéricamente como "WDWJJIR modulado", dado que se adapta en inclinación al elemento figurativo perdido por la aparición de una laguna (Guerola, 2008: 110-114).

\section{El medidor de WDWJJIR como dispositivo normativo}

\subsection{El uso de normas y estilos en las actividades grupales en clase}

Las metodologías docentes contemporáneas animan a programar acciones participativas, donde los alumnos abandonan su individualismo y sentido competitivo en favor de las actividades en grupo a través de las cuales se alcancen los objetivos de acuerdo con un plan de convergencia. En este sentido, el grupo-clase va más allá de la mera suma de sus componentes y debe aspirar a convertirse en una estructura donde se produzcan fenómenos de interacción entre los individuos que induzcan cambios positivos y avances cognitivos desde el punto de vista del aprendizaje significativo. 
Según esto y atendiendo a la definición que sobre la sociología de grupos realiza el sociólogo alemán Bernhard Schäfers (1980: 26-27), sus diferentes componentes comparten un mismo objetivo y motivación, que tienden a alcanzar a través de un proceso constante de interacción comunicativa. En este proceso se desarrolla, además, un profundo sentimiento de pertenencia al grupo, que provoca acciones solidarias en beneficio de la consecución del bien común, sustentadas en un sistema de normas; pero también, en la diferenciación de roles interdependientes que atiendan a las múltiples tareas que se puedan desempeñar.

Actuar en grupo supone, entonces, la disposición de normas y valores que marquen las líneas de acción y que refuercen esa conciencia de identidad grupal, con un clima de colaboración que además estrechará lazos afectivos (Lavilla, 2013: 3). Los cánones reglados estabilizarán, con mayor o menor margen, el orden organizativo y la estructura del grupo, marcando la trayectoria que debe seguir como unidad modular en la consecución de su meta común y, en cierto modo, mediatizando la práctica individual que queda supeditada a los modelos pautados. Este objetivo colectivo es el que debe justificar la homogeneización conductual y la cohesión grupal. Para ello, la implicación de los miembros debe ser máxima y la interacción comunicativa debe desarrollarse de una manera fluida y permanente.

\subsection{El medidor de WDWWJIR}

Considerando que, para desarrollar estrategias de colaboración, es necesario recurrir al establecimiento de normas comunes, se expone en este punto la creación por parte de cada grupo-clase de un dispositivo que sirva como patrón o modelo de muestra en los procesos de reintegración pictórica. Este instrumento estandarizado estará tipificado por los diferentes miembros de cada grupo y servirá como canon de medida referencial para llevar a cabo trabajos comunes de retoque, donde la caligrafía y calibre del WDWWJ JIR debe guardar una correspondencia genérica.

De acuerdo con este planteamiento, es importante remarcar que son los propios alumnos quienes, adoptando unas pocas normas de acción iniciales, en todo caso muy flexibles y adaptables, creen este patrón gráfico normativo. Su diseño servirá, en última instancia, para convenir y ajustar un grafismo final cuyo valor tonal se adaptate a una laguna pictórica de un supuesto práctico. Todo este trabajo cooperativo excluye cualquier situación de individualismo y competitividad, en la medida en que la consecución de los objetivos de cada miembro del grupo está correlacionada positivamente, de modo que los individuos perciben que pueden alcanzar sus propósitos en la medida en que sus compañeros de grupo también lo hagan (Deutsch, 1949; Johnson and Johnson, 2014: 841).

Las consignas iniciales son sencillas: para esta innovación se establece la creación de diferentes gruposclase, cada uno de ellos conformado por 4 componentes. Cada uno de estos equipos deberá elaborar una tabla gráfica, similar a la que se reproduce en la figura 2, teniendo en cuenta que se trata de una representación libre de color que atiende exclusivamente a una gradación en escala de grises. Con una lectura de izquierda a derecha y de arriba abajo, los diferentes campos o celdillas se ordenarán de menor a mayor valor tonal o luminosidad (Fabris y Germani, 1973: 56-58), una gradación que se conseguirá con la participación de los siguientes recursos gráficos, que hemos querido denominar "variables LCTD":

- Longitud: indica la dimensión longitudinal del trazo. Se establece que los márgenes de operatividad se encuentran entre 5 y $15 \mathrm{~mm}$.

- Contorno: estipula el grosor o ancho del perfil que, en cualquier caso, responderá a una línea fusiforme y definida con nitidez en relación con la longitud establecida.

- Translucidez: conviene el grado de transparencia u opacidad del grafismo.

- Dispersión: refiere la densidad, concentración y espaciado de elementos gráficos en el soporte espacial. 
Dentro de cada unidad de trabajo, a su vez y en correspondencia con los diferentes parámetros gráficos establecidos para la ejecución del medidor de WDWJJIR, cada integrante del grupo asumirá un rol determinado. Así pues, cada miembro tomará una variable LCTD y decidirá las características que ésta deberá tener en cada una de las celdillas que componen el medidor, teniendo siempre presente que se busca una progresión descendente de luminosidad. No obstante, estas decisiones acabarán consensuándose con el resto del grupo con el fin de detectar posibles incongruencias gráficas. De este modo, las normas procedimentales acabarán siendo implantadas internamente por los propios componentes del grupo-clase.

Por otra parte, cada individuo también se encargará de orientar a todo el grupo sobre el procedimiento más adecuado para conseguir adaptar un rayado óptimo y uniforme, según su particular rol, concretado para controlar la longitud, el contorno, la translucidez o la dispersión de los trazos dispuestos para conseguir las diferentes tramas de rayas. El objetivo final consistirá en establecer un medidor válido para cada grupoclase, donde cada integrante se haya sentido partícipe, tanto en la organización de normas, como en el proceso ejecutivo del medidor y cuyo resultado haya sido producto de un trabajo cooperativo e interdisciplinar.

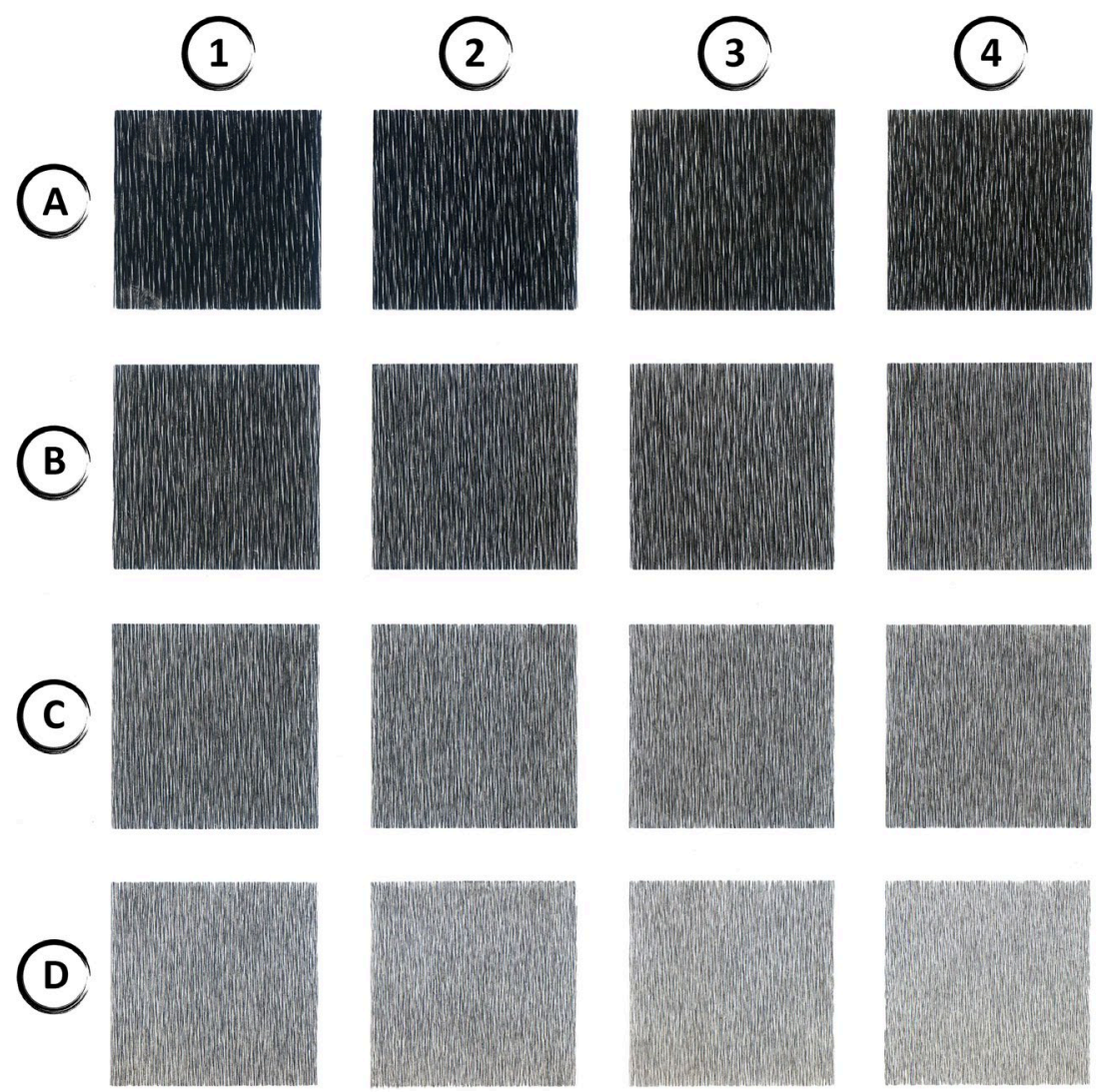

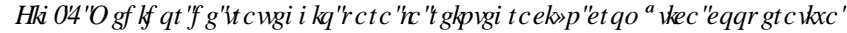

]

Para su aplicación práctica en casuísticas de obra real, el medidor de WDWJJIR ofrecerá un total de dieciséis celdillas de diverso valor tonal, conseguido con la articulación normativa de las variables LCTD. Para operar en un proceso real de reintegración cromática cooperativa, será suficiente con determinar la celdilla de referencia, simplemente haciendo valer el sistema de coordenadas cartesianas que acompaña al medidor. 
Es posible que cada grupo-clase, de acuerdo con sus propias reglas establecidas y con los criterios de funcionamiento interno, elabore un medidor de caligrafía y calibre perceptiblemente diferente. Está demostrado que, de acuerdo con las leyes de la percepción visual, la gradación en la escala de grises viene condicionada por la agudeza visual y la sensibilidad de cada individuo (Itten, 2020: 57). Pero, no obstante, obviando esta condición insalvable, lo importante es que cada grupo-clase, aun conformado por diferentes individuos, cree una herramienta de trabajo que les permita funcionar como una sola unidad modular.

\section{Otras modalidades grupales como refuerzo al aprendizaje cooperativo formal}

Con la confección del medidor de WDWJJIR a través de pequeños equipos de trabajo en el contexto del aula se han aportado evidencias de la efectividad de las dinámicas cooperativas, que viabilizan el trabajo conjunto en orden a maximizar el propio aprendizaje de cada estudiante y el de los demás componentes del grupo-clase (Johnson, Johnson and Holubec, 2013). En esta situación, en contraposición con el aprendizaje competitivo e individualista, la productividad y los logros obtenidos por cada alumno se interrelacionan con los objetivos del grupo como unidad.

También, este tipo de metodología educativa, con independencia del resultado obtenido en las actividades que se implementen y atendiendo especialmente al trayecto formativo, acaba propiciando razonamientos de mayor nivel cognitivo, con el planteamiento por parte del alumnado de propuestas y soluciones más creativas y resolutivas. Pero además, desde el punto de vista de la salud psicológica, queda demostrado que aumenta la autoestima y la confianza del individuo en sus posibilidades y las del grupo (Johnson and Johnson, 2014: 843).

La interacción en el contexto del grupo-clase favorece igualmente el desarrollo de las habilidades sociales y las relaciones personales, con todo lo que ello supone desde el punto de vista de la socialización. Impulsa la superación de conflictos (Antolín, Martín-Pérez y Barba, 2012: 7-8), fomenta la aceptación de la diversidad, la inclusión de los más desfavorecidos y ajusta ambientes alejados de conductas de rechazo o intimidación que pueden provocar aislamiento o, en última instania, el fracaso académico.

Para reforzar las cualidades del aprendizaje cooperativo formal, que supone atender a las exigencias que marca la confección del medidor como tarea principal asignada (figura 3), en este punto se propone complementar este plan con otras modalidades grupales que potencien el esfuerzo por alcanzar el logro, favorezcan las relaciones interpersonales positivas y fortalezcan el ajuste psicológico (Johnson and Johnson, 2014: 842). Todo esto será necesario en la medida en que la interdependencia positiva que surja de este sistema de aprendizaje cooperativo pueda tambalearse en el mismo momento en que alguno de los miembros del grupo pueda fracasar al quedar deteriorada, por cualquier motivo, su responsabilidad personal. $\square$

Por este peligro es por lo que las nuevas exigencias que deben implantarse en el ámbito universitario en el contexto de la convergencia europea coinciden en resaltar la importancia de una educación centrada en el sujeto que aprende (León y Latas, 2007: 271), donde se demandan nuevas competencias genéricas o transversales que, como queda reflejado en la tabla 2, solicitan destrezas tanto instrumentales, como sistémicas e interpersonales (González y Wagenaar, 2003: 81-84). Atendiendo a estas necesidades y como complemento al aprendizaje cooperativo formal, es necesario desarrollar otras dinámicas grupales que faciliten los procesos de interacción y colaboración entre los alumnos, de acuerdo con modelos de aprendizaje cooperativo informal, grupos de base y debates de controversia constructiva (Johnson and Johnson, 2014: 842). 


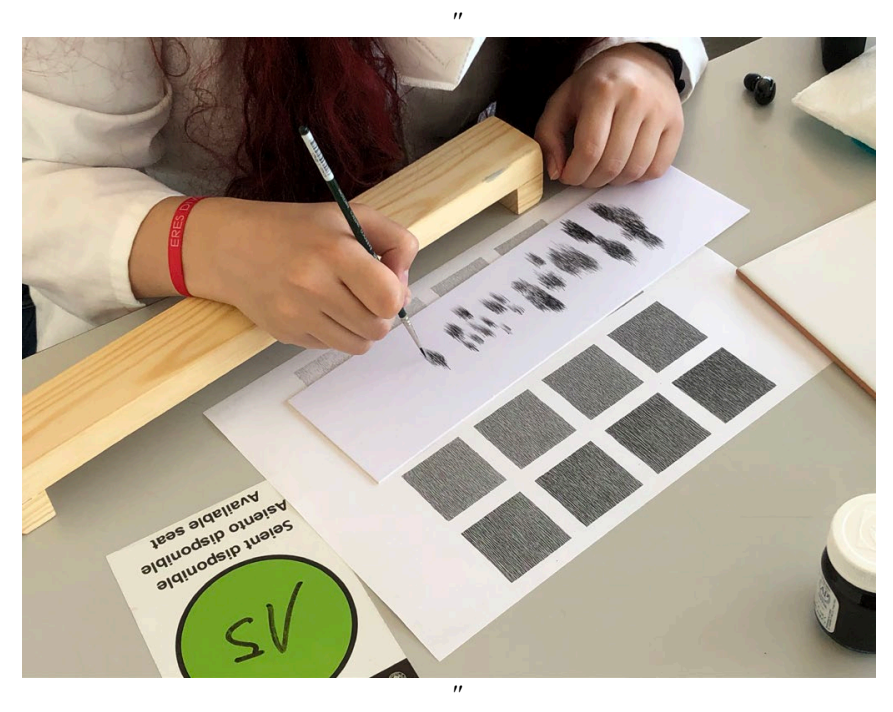

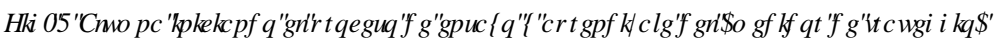

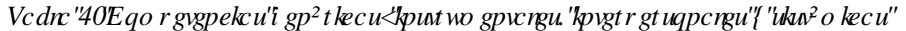

\begin{tabular}{|c|c|c|}
\hline Competencias instrumentales & Competencias interpersonales & Competencias sistémicas \\
\hline capacidad de análisis y síntesis & capacidad crítica y autocrítica & $\begin{array}{l}\text { capacidad de aplicar los } \\
\text { conocimientos en la práctica }\end{array}$ \\
\hline $\begin{array}{c}\text { capacidad de organizar y } \\
\text { planificar }\end{array}$ & trabajo en equipo & habilidades de investigación \\
\hline $\begin{array}{c}\text { conocimientos generales } \\
\text { básicos }\end{array}$ & habilidades interpersonales & capacidad de aprender \\
\hline $\begin{array}{l}\text { conocimientos básicos de la } \\
\text { profesión }\end{array}$ & $\begin{array}{l}\text { capacidad de trabajar en un } \\
\text { equipo interdisciplinar }\end{array}$ & $\begin{array}{l}\text { capacidad para adaptarse a } \\
\text { nuevas situaciones }\end{array}$ \\
\hline $\begin{array}{c}\text { comunicación oral y escrita en } \\
\text { la propia lengua }\end{array}$ & $\begin{array}{c}\text { capacidad para comunicarse con } \\
\text { expertos de otras áreas }\end{array}$ & $\begin{array}{c}\text { capacidad para generar nuevas } \\
\text { ideas (creatividad) }\end{array}$ \\
\hline $\begin{array}{c}\text { conocimiento de una segunda } \\
\text { lengua }\end{array}$ & $\begin{array}{c}\text { apreciación de la diversidad y } \\
\text { multiculturalidad }\end{array}$ & liderazgo \\
\hline $\begin{array}{c}\text { habilidades básicas de manejo } \\
\text { del ordenador }\end{array}$ & $\begin{array}{l}\text { habilidad de trabajar en un } \\
\text { contexto internacional }\end{array}$ & $\begin{array}{l}\text { conocimiento de culturas y } \\
\text { costumbres de otros países }\end{array}$ \\
\hline $\begin{array}{l}\text { habilidades de gestión de la } \\
\text { información }\end{array}$ & compromiso ético & $\begin{array}{c}\text { habilidad para trabajar de forma } \\
\text { autónoma }\end{array}$ \\
\hline resolución de problemas & & diseño y gestión de proyectos \\
\hline \multirow[t]{2}{*}{ toma de decisiones } & & $\begin{array}{c}\text { iniciativa y espíritu } \\
\text { emprendedor }\end{array}$ \\
\hline & & $\begin{array}{c}\text { preocupación por la calidad } \\
\text { motivación de logro }\end{array}$ \\
\hline
\end{tabular}

Fuente: González, J. y Wagenaar, R. (2003: 83-84) 


\subsection{El aprendizaje cooperativo informal}

Mas allá de las tareas asignadas en los grupos cooperativos para cumplir con los objetivos específicos marcados en correspondencia con los contenidos de la asignatura, la cooperación informal, con las múltiples variantes que ofrece (Karge $H W D O, 2011$ ), refuerza la cohesión grupal y funciona como un andamiaje sobre el que seguir avanzando. Este soporte, especialmente desde el punto de vista de la andragogía o la ciencia que enseña a los adultos a aprender (Knowles $H W D O, 2020: 18-50)$, robustece los lazos interpersonales frente a cualquier amenaza que pueda desestabilizar la unión.

Después de las primeras explicaciones por parte del profesor para la elaboración del medidor, con la creación de grupos y la asignación de roles de acuerdo con las variables LCTD, se invitará a que, en un primer momento y de manera provisional, con un encuentro expeditivo y eficaz, los estudiantes se agrupen por roles.

En estos grupos iniciales, no definitivos o informales, se desarrollará un método muy próximo al $7 \mathrm{KIQN}$ 3 DU6KDH (TPS) (Lyman, 1987; Lange, Costley and Han, 2016: 262). Para el desarrollo de esta estrategia TPS cada grupo informal estará constituido por individuos con el mismo rol, que debatirán sobre las características que deberá tener el trazo de WDWJJIR de acuerdo con la variable LCTD que representan (longitud, contorno, translucidez o dispersión). En estas primeras reuniones es imprescindible la presencia del profesor que, en cada uno de los grupos, irá dando respuesta a sus problemáticas y preguntas a través de una evaluación formativa. Los pasos que se requieren para esta estrategia TPS, como actividad previa a la elaboración del medidor de WDWWJJIR y con una clara progresión hacia niveles cognitivos superiores (Bloom $H M D O, 1956)$, quedan recogidos en la tabla 3.

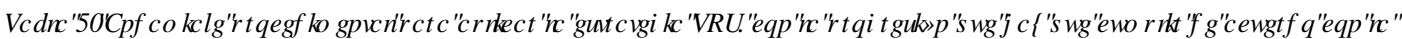 GP HQMYQGHIRVSLRFHRVFRUQUWRV}

\begin{tabular}{ll}
\hline Fases progresivas & \multicolumn{1}{c}{ Actividad estratégica } \\
\hline Paso 1. Recordar & $\begin{array}{l}\text { El profesor pregunta a los estudiantes acerca de las } \\
\text { cualidades que debería tener el WDWJJIR, en relación con } \\
\text { la variable LCTD asignada a cada alumno }\end{array}$ \\
Paso 2. Entender & $\begin{array}{l}\text { Los estudiantes piensan individualmente sobre cómo } \\
\text { responder a la pregunta }\end{array}$ \\
Paso 3. Aplicar & $\begin{array}{l}\text { Los estudiantes toman notas y diseñan trazos de prueba } \\
\text { con diferentes propuestas gráficas }\end{array}$ \\
Paso 4. Analizar & $\begin{array}{l}\text { Los estudiantes con el mismo rol forman grupos y } \\
\text { comparten sus ideas y planteamientos }\end{array}$ \\
Paso 5. Evaluar & $\begin{array}{l}\text { Las similitudes y diferencias de las diversas propuestas } \\
\text { son anotadas, debatidas y evaluadas en grupo }\end{array}$ \\
Paso 6. Crear & $\begin{array}{l}\text { Se crea una respuesta final como resultado cooperativo, } \\
\text { que se expone a toda la clase }\end{array}$ \\
\hline
\end{tabular}


De este modo, previamente a la incorporación definitiva en grupos de trabajo cooperativo para la ejecución del medidor, cada componente del grupo habrá experimentado una potente formación colaborativa en referencia a su rol, de modo que controlará y normalizará su variable LCTD en su grupo de trabajo, como experto previamente instruido que ha filtrado la información básica para extraer los puntos clave sobre los que trabajar.

En definitiva y de acuerdo con todo esto, durante los años en los que se ha venido aplicando la innovación, se establecieron 4 grupos informales, compuestos por 8 estudiantes cada uno, que compartieron y trabajaron sobre una misma variable LCTD. Sus reuniones se desarrollaron siempre al inicio de la clase, durante la primera media hora y a lo largo de los dos primeros días de trabajo. En esta sesgunda sesión, además, se reservó un tiempo al final de la reunión informal para que cada grupo expusiera sus conclusiones a toda la clase. Su objetivo fue el de especificar por grupos de componentes con idéntico rol qué características debería tener el rayado efectuado en los procesos de reintegración cromática.

\subsection{Los grupos de base cooperativos}

Paralelamente a la consecución de las tareas principales, en este caso la elaboración del medidor de WWWJJIR, es conveniente que dentro de los grupos de trabajo cooperativo formal se cuiden determinados aspectos funcionales. El engranaje del grupo debe estar perfectamente articulado y cada componente debe velar para que se active y opere como un todo único. Ninguna pieza puede verse afectada y, con ello, perturbar el ritmo del equipo.

Por esto, el grupo de cooperación formal debe convertirse en algunos momentos en lo que se conoce como un grupo de base (Johnson and Johnson, 2014: 842). En el caso que nos ocupa, el grupo invertirá unos pocos minutos antes o después de cada sesión de trabajo para atender, evaluar y resolver cualquier tipo de problemática que afecte a alguno de sus integrantes y que pudiera ralentizar el correcto funcionamiento del conjunto.

En este momento, donde además el profesor actuará como facilitador del aprendizaje (Carnap, 2007), es cuando se tratarán cuestiones referentes al progreso académico individual, a las faltas de asistencia e impuntualidad o, al modo en que tender la mano o poner al día a los estudiantes ausentes, irregulares o de ritmo más pausado. Las interconexiones sociales en este punto se harán más evidentes y las influencias constructivas proporcionarán estímulos favorables y suscitarán la motivación. Conforme a la tabla 1, durante los cursos que se aplicó esta innovación se programaron estas reuniones al final de la tercera sesión de trabajo y al principio de la quinta sesión de trabajo, con una duración aproximada de 15 minutos. En estas conversaciones de base se expusieron y trataron problemáticas como las que se especifican a continuación:

- ¿Todos los componentes del grupo han asistido a clase con regularidad y puntualidad?

- En el caso de haberse producido faltas de asistencia o impuntualidad por parte de algún alumno ¿cómo ha repercutido esta circunstancia a nivel individual y en el ritmo de trabajo del grupo?

- ¿Existe algún componente que muestre un ritmo de trabajo irregular o descompasado en relación con el resto de sus compañeros? ¿cuáles son las causas y cómo sería posible ajustar estos desfases?

- ¿ ¿Se detenta algún tipo de problema académico o personal en alguno de los miembros del equipo que pueda influir de alguna manera en el ritmo normal de trabajo?

El objetivo final buscará adecuar una participación equitativa por parte de todos los activos del conjunto. En el caso de que esto no ocurriera existiría una mayor posibilidad de que los estudiantes que queden descolgados del grupo, técnicamente conocidos como viajeros gratuitos o IUHUGHV(Johnson \& Johnson, 1994; Onwuegbuzie, Collins y Jiao, 2009; Lange, Costley and Han, 2016: 264), contagien su situación hasta conseguir un estado de holgazanería o pereza social, muy perjudicial para el progreso colectivo.

(c)) BY-NC-ND 2021, Universitat Politècnica de València

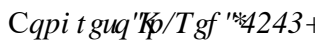




\subsection{Las controversias constructivas}

De manera implícita, el aprendizaje cooperativo está fundamentado en la resolución de conflictos, siempre presentes con mayor o menor manifestación en este tipo de metodologías grupales de colaboración. Por esto, es difícil concebir un grupo-clase donde impere exclusivamente un ambiente de conformidad, aquiescencia y consenso unánime.

Los acuerdos integradores como fruto de una cooperación constructiva frente a situaciones donde convergen diferentes puntos de vista, con opiniones disyuntivas o incompatibles, captan la atención y despiertan el interés de los alumnos, potenciando la creatividad y la innovación en la búsqueda de consensos (Johnson, 2016; Johnson and Johnson, 2014: 842). Por esto, las sistemáticas docentes basadas en el conflicto sociocognitivo deben potenciarse con el claro objetivo de incentivar el desarrollo intelectual (Gavilán, 2009: 134-139).

Considerando esta necesidad, durante las sesiones de trabajo en clase, tanto en los grupos cooperativos formales como informales, el profesor provocará intencionadamente la confrontación en el momento en que los alumnos se encuentren debatiendo acerca del calibre y caligrafía del trazo al que debe atender el medidor de WDWIJIR. Actuará como agente catalizador de conflictos, invitando incluso a que los propios componentes del grupo participen en esta dinámica, asumiendo la figura del "abogado del diablo" (Sánchez, 2011).

De este modo, en las reuniones de los grupos informales, programadas para que se desarrollen al inicio de las dos primeras sesiones de trabajo, el profesor considerará las dificultades y contradicciones que puedan generarse en relación con la variable LCTD que esté trabajando cada grupo. En este momento lanzará preguntas para empezar debates o diálogos socráticos que tengan como finalidad ajustar un consenso en cada grupo informal, que aparezca como consecuencia de la controversia constructiva que se establezca al responder a las siguientes cuestiones:

- Grupo informal L: ¿qué longitud debe tener el trazo en cada una de las celdas que componen el medidor de WDWAJJIR? ¿se establece una gradación en este parámetro que está en consonancia con los diferentes valores tonales del medidor?

- Grupo informal C: ¿qué grosor de línea debe definirse en las diferentes celdillas del medidor? ¿el grosor del rayado guarda consonancia con la longitud del trazo?

- Grupo informal T: ¿qué grado de transparencia u opacidad debe proyectarse en cada una de las celdas? ¿es posible establecer un porcentaje de luminosidad o valor tonal que defina objetivamente cada celda?

- Grupo informal D: ¿qué densidad, concentración y espaciado, en cualquier caso con una trama uniforme de líneas, debe regir la estructura gráfica de cada celdilla?

\section{Resultados}

Como producto del trabajo cooperativo en la elaboración del medidor de WDWJJIR se ha revelado una significativa interdependencia positiva, lo que supone la consecución de un bien o meta común solo alcanzable con el trabajo conjunto y en consonacia con la suma de los logros individuales. El microcosmos que supone el grupo-clase puede entenderse como un reflejo en miniatura de la sociedad actual, cuyos retos y conflictos demandan soluciones de cooperación global.

La aplicación de esta innovación docente ha comportado resultados satisfactorios, en la medida en que los alumnos han propuesto soluciones finales creativas y originales, que han confluido gracias a la búsqueda de puntos de encuentro entre las diferentes voces participantes. El establecimiento de roles y la distribución 
de tareas conceden al alumno responsabilidades derivadas de su función, de modo que su compromiso e implicación en el grupo aumentan en la medida en que los propios componentes se encargan de establecer las normas de funcionamiento y las pautas procedimentales.

El aprendizaje cooperativo, potenciado con actividades como la propuesta en esta contribución, es necesario para afrontar todas las problemáticas que se derivan de la interdependencia global en todos los niveles y disciplinas. Este escenario creará hábitos beneficiosos que, si además se potencian con círculos de actividad informal o grupos de base, favorecerán el desarrollo de las competencias genéricas del individuo y su conducta prosocial en un contexto más amplio de socialización.

Las evidencias para la valoración de esta innovación se establecen, por un lado, atendiendo a los resultados positivos de las encuestas de alumnado, que suponen un sistema efectivo para el control de la calidad docente por parte de los estudiantes, actores directos del proceso de aprendizaje. A la vista de los resultados obtenidos se concluye que la satisfacción general ha sido óptima, con una media por encima de 9 sobre 10 en este tramo de la asignatura. Entre los puntos que se relacionan, que atienden al papel del profesor en la innovación y que se han considerado con mayor valoración cabe citar:

- Tiene una buena programación del ritmo de las clases y del tiempo dedicado a cada tema

- Emplea metodologías y actividades en el desarrollo de la asignatura que ayudan a aprender al alumnado

- Contribuye a crear un buen clima de trabajo y anima al alumnado a participar durante el desarrollo de la asignatura

- Consigue motivar al alumnado y despertar el interés por la asignatura

Por otro lado, también fue cumplimentado por parte del alumnado un cuestionario de evaluación conformado por el profesor y que fue organizado específicamente para responder a cuestiones relativas al desarrollo de esta unidad didáctica de la asignatura, con unos resultados satisfactorios. Esta valoración vuelca resultados muy positivos, especialmente relacionados con las siguientes cuestiones:

- $\quad$ Aumento de la satisfacción del alumno

- Fomento del trabajo en grupo

- Aprendizaje más activo y experiencial

- Interacción con el profesor y compañeros más frecuente y positiva

- Desarrollo de competencias transversales y educación prosocial

Por último, el acto de evaluación sumativa para balancear de manera fiable los logros obtenidos al final del proceso de aprendizaje ha puesto de manifiesto la correcta asimilación de los contenidos de la asignatura por parte del alumnado y el cumplimiento de los objetivos planteados de inicio.

\section{Conclusiones}

El aprendizaje cooperativo, que se presenta en este estudio, trasciende respecto de los métodos más tradicionales y académicos para implementar, de acuerdo con los requerimientos del Espacio Europeo de Educación Superior (EEES), métodos docentes que fomenten en el alumnado el desarrollo del sentido crítico, de acuerdo con una visión interpersonal tolerante y de crecimiento mutual. En este sentido, resulta trascendental fomentar en el aula hábitos de participación colaborativa que ayuden a avanzar hacia altos niveles cognitivos a la vez que se promueve el trabajo en la tolerancia, el respeto y la solidaridad.

A través de la elaboración en grupo de una herramienta de caligrafía y calibre que sirva para consensuar la tipología gráfica que ha de seguir el WDWJJIR en los procesos de reintegración cromática en obras de arte 
se pretende crear en el alumno hábitos de trabajo cooperativo. La finalidad última de establecer estos automatismos es poder extrapolar estas conductas a cualquier ámbito de su futuro profesional y social. Esta metodología de trabajo es clave en la actualidad, tanto en los sistemas de gestión profesional y empresarial, como en las organizaciones políticas o gubernamentales, donde se reclaman soluciones que emerjan como resultado de la articulación solidaria de esfuerzos.

\section{Referencias}

ANTOLÍN, Á., MARTíN-PÉREZ, G. y BARBA, J. J. (2012). "El aprendizaje cooperativo para la mejora de la socialización y

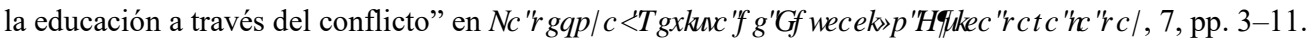

BALDINI, U. (1981). 7HRUD GHD UHMDXLR H XQLML GL P HRRRRID (Vol. 2). Firenze: Nardini Editore - Centro Internazionale del Libro.

BALDINI, U. (1978). 7HRUD GHD UHMDXLR H XQLMD GL P HRRRRJID (Vol. 1). Firenze: Nardini Editore - Centro Internazionale del Libro.

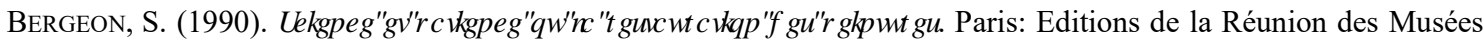
Nationaux.

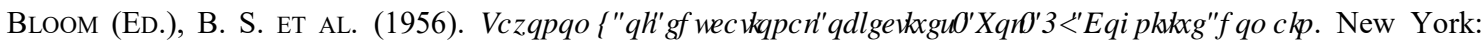
Longmans.

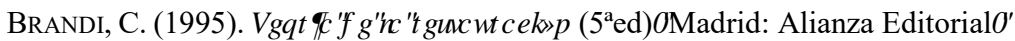

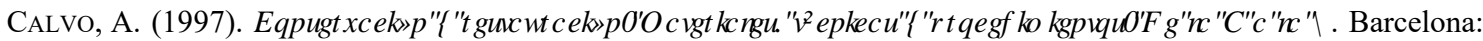
Ediciones del Serbal.

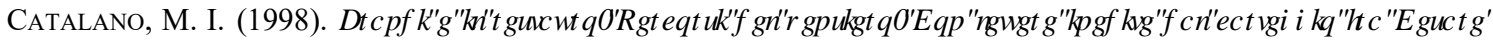
\%DQCUH( QUFRT9DCOFFKL Firenze: $\mathbb{N}$ ardini Editore, pp. 42-54.

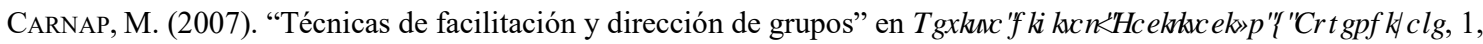
pp. $1-4$.

DeUTSCH, M. (1949). “A theory of cooperation and competition” en + XPDQ5 HDURQV, 2, pp. 129-152.

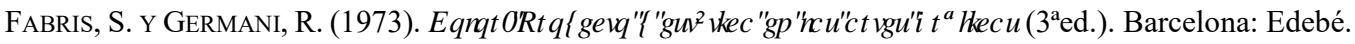

Gavilán, P. (2009) "Aprendizaje cooperativo. Papel del conflicto sociocognitivo en el desarrollo intelectual. Consecuencias pedagógicas" en 5HLWDHSDRRDTGHSHDJ RJ tD, 242, pp. 131-148.

GONZÁLEZ, J., WAGENAAR, R. (COORD.) (2003) 7XQQJ IHLXFDMRQDOMUXFWXHUIQ( XURSH Bilbao: University of Deusto.

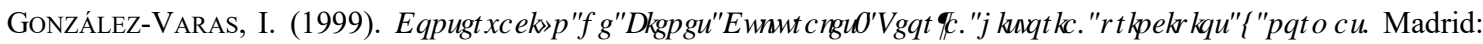
Ediciones Cátedra.

Guerola Blay, V. (2008). "Ideología y metodoclogía en la reintegración de lagunas, de la técnica del QHUDQIIDUHal retoque pleno pasando por los sitemas ópticos de texturización basados en los IIDP HQWHWDWJJLde Cesare Brandi"

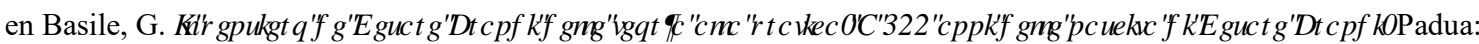
Il Prato Editore, pp. 110-114.

ITTEN, J. (2020). ( ODUATGHARRU Barcelona: Editorial Gustavo Gili.

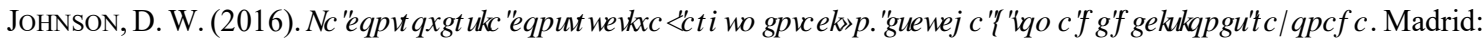
Ediciones SM.

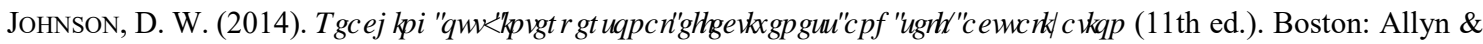
Bacon. 
Johnson, D. W. \& Johnson, R. T. (2014). “Cooperative Learning in 21 st Century. [Aprendizaje cooperativo en el siglo XXI]” en \$ QDOMKGH3 UFR(RJ tDII QQDORRI[3 K FKRRJ I, 30(3), pp. 841-851.

Johnson, D. W., Johnson, R. T. \& Holubec, E. J. (2013). \&RRSHDMRQ IQ WKH\&QWRRP (9th ed.). Edina, MN: Interaction Book Company.

Johnson, R. T. \& Johnson, D. W. (1994). “An overview of cooperative learning” in Thousand, J.,Villa, R. \& Nevin,

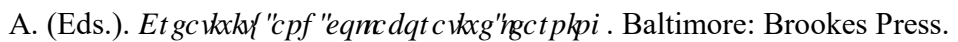

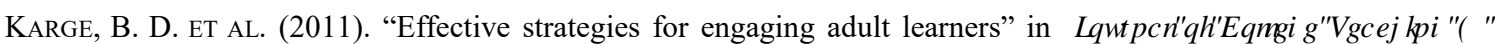
/ HDQQU, 8(12), pp. 53-56.

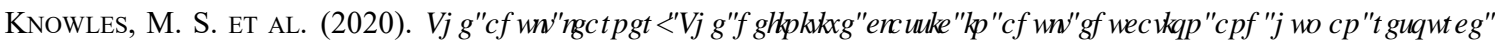
GHHIRSP HQW(9th Edition). New York: Routledge.

LANGE, C., Costley, J. \& HAN, S. L. (2016). "Informal cooperative learning in small groups: the effect of scaffolding on participation" in , WXHUIQ( GXFDURQDO5 HMHFK, 26(2), pp. 260-279.

LAVILLA, L. (2013). "Dinámica del grupo clase” en 5 HLWWGH\&QDMKLMRUD, 5, pp. 1-10.

LEÓN, B. y LATAS, C. (2007). "La formación en técnicas de aprendizaje cooperativo del profesor universitario en el contexto de la convergencia europea" en 5HLWWGHSVFRGG FUFD, 12(2), pp. 269-277.

LyMAN, F. (1987). "Think-Pair-Share: An expanding teaching technique” in $0 \$ \$ \square \&$,( $\square \& R R S H D M H H 1$ HV V, 1, pp. 1-2.

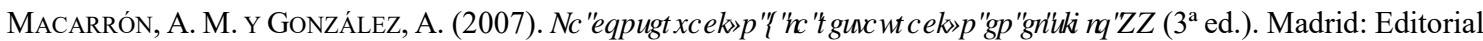
Tecnos.

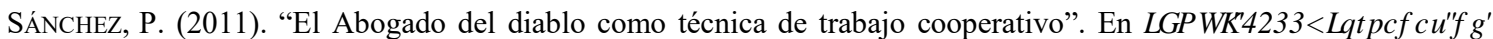

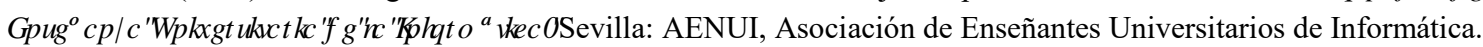
$127-134$.

SCHÄFERS, B. (1980). "Evolución de la sociología de grupos e independencia del grupo como formación social" en Schäfers, B. , QURGXFFY QDIDUVFIR(RJ LDCHJUXSRV. Barcelona: Herder, pp. 25-40.

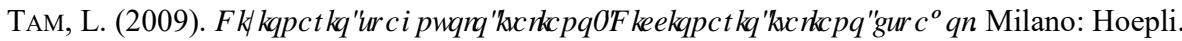

